arterial line in selected cases, but in our opinion it is neither necessary nor justifiable to insert routinely intra-arterial lines in all patients admitted to a general medical intensive care unit.

Flexibility is the essence of modular monitoring apparatus. It allows the clinician to decide which physiological variables he wishes to monitor, and he should never regard such a system as a "panindicator" unit. Therefore each separate system must be reliable in its own right, and reliability in the clinical environment can be assessed only by intensive study in a clinical situation. The technique we used seems to give a useful indication of whether a particular system is suitable for routine clinical use. Moreover, we would suggest that to be of value in hospital conditions an automatic monitor needs to be at least $90 \%$ reliable.
We would like to acknowledge the unstinted co-operation of the nursing staff of Starling Ward; Mr. A. J. Solman, of the National Institute for Medical Research; and Mr. A. S. Velate, of the T.E.M. Engineering Company.

This work was supported by the Medical Research Council.

\section{References}

Clark, T. J. H., Collins, J. V., Evans, T. R., and Tweedily, K. (1971). British Medical fournal, 1,158

Crockett, A. S., and Barr, A. (1965). British Medical fournal, 2, 1173.

De Dobbeleer, jun., G. D. P. (1965). World Medical Electronics and Instrumentation, 3,1222 .

Rawles, J. M., and Crockett, G. S. (1969). British Medical fournal, 3, 707.

Wolff, H. S. (1966). British Hospital fournal and Social Service Review, 76, 1991 .

\title{
Monitoring Cerebral Function: Clinical Experience with New Device for Continuous Recording of Electrical Activity of Brain
}

\author{
PAMELA F. PRIOR, D. E. MAYNARD, P. C. SHEAFF, B. R. SIMPSON, L. STRUNIN, \\ E. J. M. WEAVER, D. F. SCOTT.
}

British Medical fournal, 1971, 2, 736-738

\begin{abstract}
Summary
A device, the cerebral function monitor, provides a continuous record of the electrical activity of the brain occurring at frequencies from 2 to $15 \mathrm{~Hz}$. It is relatively cheap, portable, and easy to use and interpret. The apparatus has proved of value in three circumstances: firstly, when the cerebral circulation is likely to be vulnerable during open heart surgery; secondly, as a measure of recovery or deterioration following brain damage or drug overdose; and thirdly, where information about more physiological changes in cerebral function is required, for instance when testing anaesthetic and hypnotic drugs.
\end{abstract}

\section{Introduction}

Continuous monitoring of cerebral activity may be of particular value in two situations. Firstly, when the cerebral circulation is likely to be vulnerable, for instance during open cardiac surgery, and secondly, as a measure of recovery or deterioration following brain damage or in drug induced coma. The use of electroencephalographic (E.E.G.) apparatus for examining the electrical activity of the brain for long periods is complex and

The London Hospital, London E1 1BB

PAMELA F. PRIOR, M.B., Medical Assistant, E.E.G. Department D. E. MAYNARD, M.PHIL., Research Engineer, E.E.G. Departmen

P. C. SHEAFF, M.R.C.P., Registrar, E.E.G. Department (At present Senior Registrar, Department of Clinical Neurophysiology, St. Bartholomew's Hospital, London EC1A 7BE)

B. R. SIMPSON, D.PHIL., F.F.A. R.c.s., Professor, Anaesthetics Unit L. STRUNIN, M.B., F.F.A. R.C.S., Senior Lecturer and Consultant Anaesthetist, Anaesthetics Unit

E. J. M. WEAVER, F.R.C.s., Surgeon, Department of Cardiothoracic Surgery

D. F. SCOTT, M.R.C.P., D.P.M., Consultant-in-Charge, E.E.G. Department costly in terms of specialized staff and apparatus (Feldman and Ellis, 1967; Haider et al., 1971). Equipment aimed at monitoring brain activity has been part of a system for providing servo-anaesthesia (Bickford, 1949) or used to indicate the depth of anaesthesia (Efuni et al., 1965); none has been primarily used for providing information about the state of cerebral activity.

A cerebral function monitor (C.F.M.) has recently been introduced (Maynard et al., 1969) as a result of experience at the London Hospital of E.E.G. recording in the operating theatre during cardiac surgery (Fischer-Williams and Cooper, 1964) and in the intensive therapy unit (Prior and Volavka, 1968; Binnie et al., 1970). The C.F.M. is designed to obtain a simple, continuous assessment of cerebral function over long periods without the need for skilled attention. This communication reports our use of the C.F.M. in patients following cardiorespiratory arrest and during open heart surgery. Studies have been undertaken in close parallel with conventional E.E.G. recordings; these have been carried out every 24 to 48 hours on patients in the intensive therapy unit and continuously throughout most of the operations on the patients undergoing cardiac surgery. On occasions the C.F.M. has been used alone during cardiac surgery to see whether it is of value to the anaesthetist without the aid of an E.E.G. technician or an electroencephalographer.

\section{Methods and Materials}

The technical details of the C.F.M.* have been described (Maynard et al., 1969). Briefly, cerebral activity is picked up by two conventional silver and silver chloride electrodes attached with collodion to the parietal regions on both sides of the head after preliminary separation of the hair and cleansing of the scalp. This site was chosen to reduce muscle and movement artefact and to minimize interference during nursing procedures. The output of the monitor is seen as two traces on a hot wire recorder. The first trace gives a continuous record of the

*Available from Devices Limited, Welwyn Garden City, Herts. 
mean amplitude and variation of the cerebral activity, this being first passed through a trap to eliminate radiofrequency interference and filtered to reject frequencies outside the range $2-15 \mathrm{~Hz}$, with very high rejection of $50 \mathrm{~Hz}$. Precaution is also taken to exclude capacitative interference caused by movement of electrode leads and articles of statically charged clothing. Thus most non-E.E.G. or "artefactual" potentials are eliminated, whether of biological or of external origin. The second trace shows both the state of conductivity of the electrodes and any blocking, overloading, or malfunction of the amplifier. The second trace therefore indicates whether the cerebral trace is unreliable due to artefact such as diathermy, heavy mains interference, or movement of the electrodes.

After calibration an appropriate paper speed is selected for the chart recorder, $2.5-9 \mathrm{~cm}$ hour for recordings in the intensive therapy unit and $36 \mathrm{~cm} /$ hour for theatre recording. (This contrasts with a paper speed of $1,080 \mathrm{~cm} /$ hour for conventional E.E.G. recordings.) The apparatus runs without attention. Any procedure carried out on the patient-for example, turning or the administration of drugs-may be marked directly on the tracing.

Upward or downward movement of the slope of the tracing on the C.F.M. corresponds to increase or decrease in the amount of cerebral activity, and, similarly, gradations in width and variability of the tracing indicate the character of cerebral activity. The Table shows the comparative merits of the C.F.M. in relation to the conventional E.E.G. apparatus.

Comparison of C.F.M. and Conventional E.E.G. Apparatus

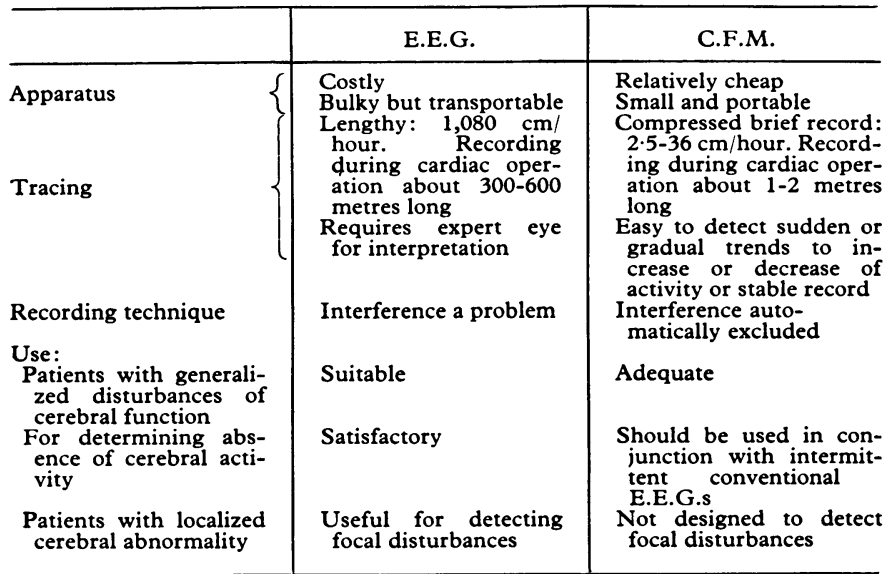

\section{Clinical Applications}

Eighty-six patients have been monitored, 49 during cardiac surgery with cardiopulmonary bypass and 37 following resuscitation after cardiorespiratory arrest. The age of the patients ranged from 3 to 73 years, and monitoring in the intensive therapy unit was continuous for periods of up to five weeks.

Patients Monitored during Cardiac Surgery with Cardiopulmonary Bypass.-The C.F.M. has proved of value in two main circumstances during cardiac surgery. Firstly, to indicate

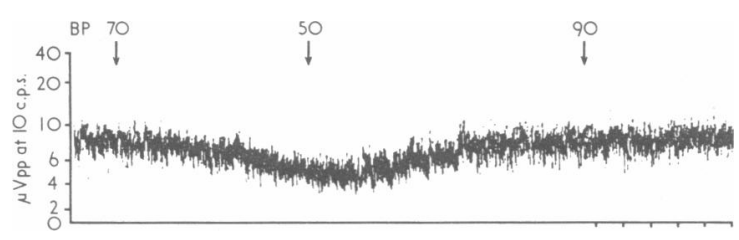

FIG. 1-Episode of hypotension producing a transient reduction in the amount of cerebral activity is shown. The marks on the baseline are at one-minute intervals. inadequate cerebral perfusion in association with hypotension (Fig. 1), periods of ventricular fibrillation (Fig. 2), and innominate and common carotid steal in relation to the position of the aterial cannular in the arch of the aorta. At these times the C.F.M. showed a decrease in the level of activity, which fell to baseline when there was complete failure of cerebral perfusion.

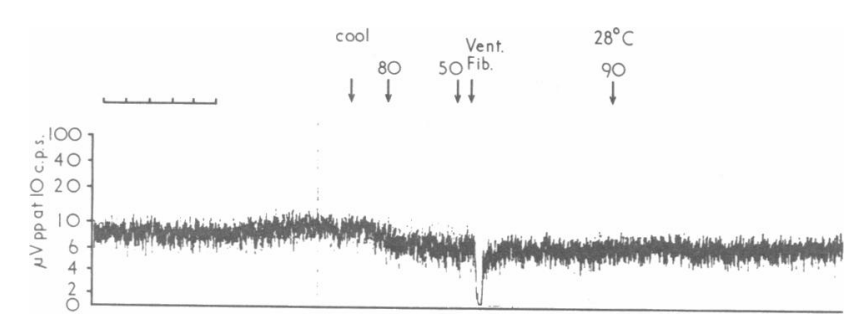

FIG. 2-Short-lived episode of ventricular fibrillation which occurred during cooling is shown in a 59-year-old man during an operation for aneurysm of the ascending aorta and aortic regurgitation. A sudden drop to baseline lasting 20 seconds coincided with a similar period of electrical silence in the conventional E.E.G. Blood pressures are indicated. The time marker shows one-minute intervals.

Prompt restoration by therapeutic procedures of a reasonable level of cerebral activity on the C.F.M. was not associated with postoperative evidence of cerebral damage. However, patients in whom the level remained low did show neurological abnormality. Secondly, in the absence of clinical evidence, alterations in the level of anaesthesia may be observed when beginning cardiopulmonary bypass (Fig. 3) or at other times during the procedure (Fig. 4).

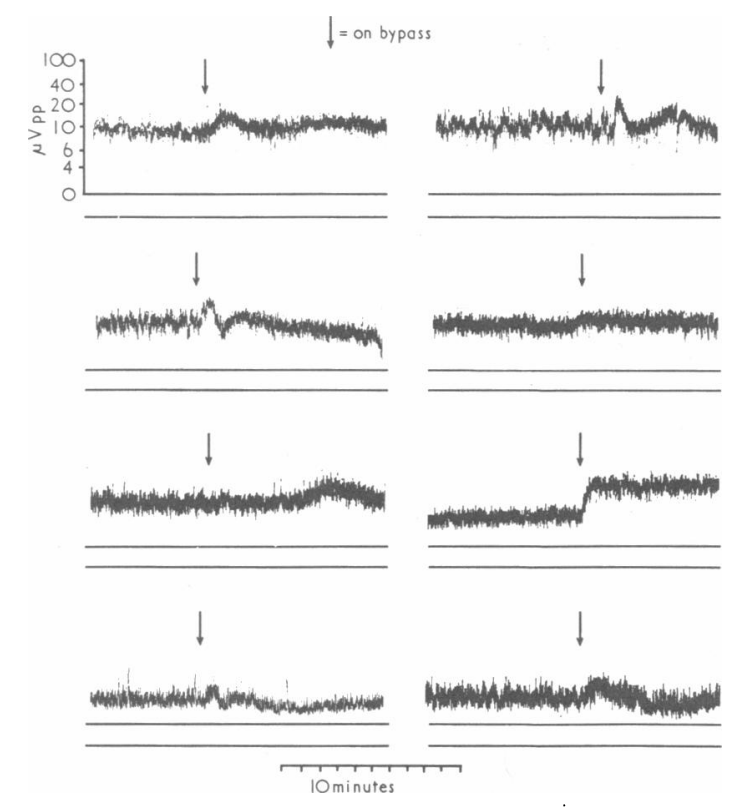

FIG. 3-Examples of the C.F.M. tracing at the beginning of cardiopulmonary bypass in eight different patients are given. The arrows indicate the onset of bypass. The calibration is the same for each tracing.

Patients Monitored after Cardiorespiratory Resuscitation.The major problem for the clinician in a patient who fails to recover consciousness after cardiorespiratory arrest is assessment of cerebral function. As has been mentioned previously, conventional E.E.G. recording can only be undertaken intermittently; it requires portable apparatus and skilled staff and is time-consuming and expensive. In contrast, since the C.F.M. provides a continuous, if limited, record of cerebral activity, 

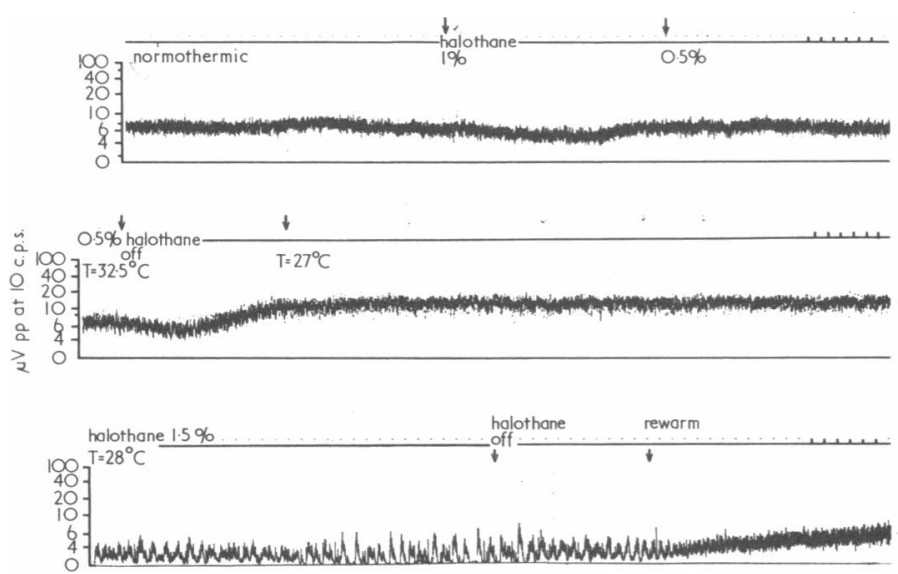

FIG. 4-Appearances in the C.F.M. tracing in relation to halothane administration, moderate hypothermia separately and in combination are shown. The upper two tracings show the relatively slight effects of 0.5 and $1 \%$ halothane in a normothermic subject and of cooling to $27^{\circ} \mathrm{C}$ when halothane is not being administered, the lower trace a "saw-tooth" effect seen when halothane $1.5 \%$ is combined with cooling to $28^{\circ} \mathrm{C}$. The marker indicates one-minute intervals.

improvement or deterioration is readily observed (Fig. $5 \mathrm{~B}$ and C); also sudden changes, for instance during seizures (Fig. 6), are immediately apparent. Continuous confirmation of near total absence of cerebral activity over a long period of time (Fig. $5 \mathrm{~A}$ ) and between conventional E.E.G. recordings may enable the clinician to predict with greater confidence that

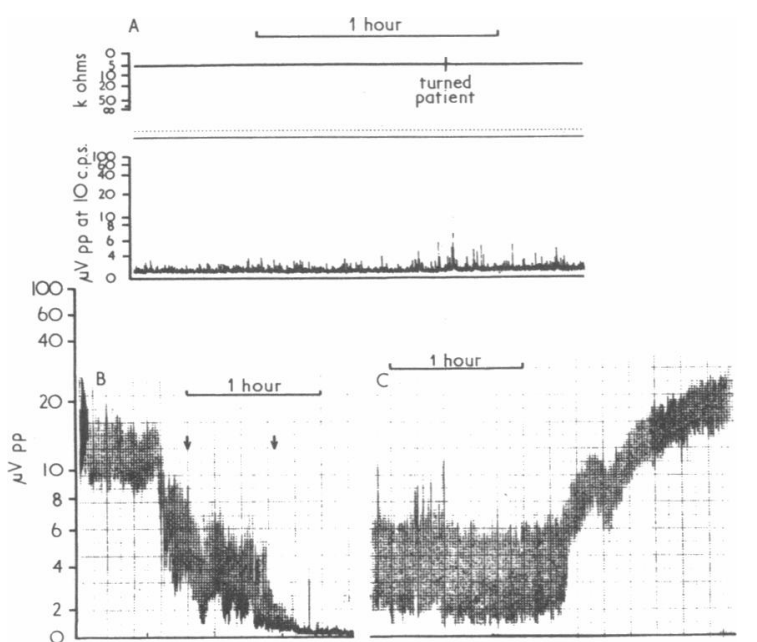

FIG. 5-Examples of typical appearances of C.F.M. tracings in patients after resuscitation are shown. (A) A continuous near zero-level tracing in a patient after cardiac arrest; note the indication of artefact on the upper (impedance monitor) tracing at the time of a movement. (B) A fall in C.F.M. level associated with an hypotensive episode and cardiac arrest. (C) A rise in C.F.M. level in a patient deeply comatose after a barbiturate overdose and respiratory arrest. The rise preceded clinical evidence of improvement by many hours.

irreversible brain damage has occurred. Eight patients with such isoelectric records and sustained zero-level C.F.M. tracings were subsequently found at neuropathological examination to have suffered from severe anoxic cerebral damage. These patients showed either widespread selective neuronal necrosis or total cortical necrosis. Correlation has also been demon-

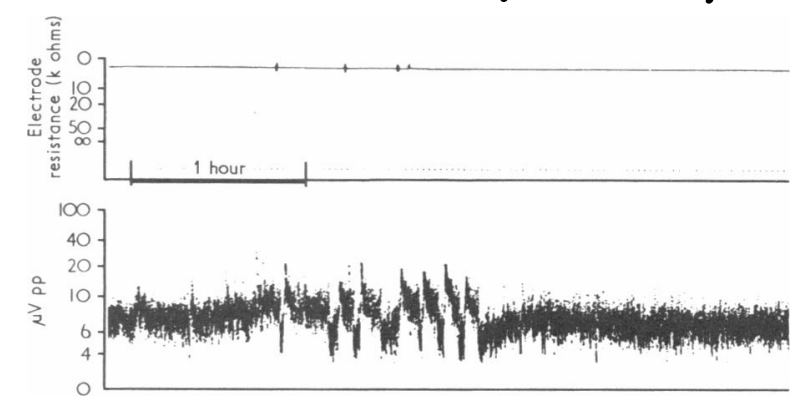

Fig. 6-Appearance of the C.F.M. at the time of seizures in a 17-year-old patient lightly comatose after cardiac arrest is indicated. The small marks the occurrence of seizures.

strated neuropathologically with less severe anoxic brain damage and reduced levels of activity on the C.F.M.

Other Applications.-As well as the two main uses outlined above, and because the C.F.M. is relatively cheap, portable, and easy to use and interpret, it is also of value in instances where information about other more physiological changes in cerebral function is required. For example, it has been possible, in addition to timing the onset of sleep, to correlate changes in the C.F.M. trace with rapid eye movement and non-rapid eye movement stages of sleep (Dement and Kleitman, 1957). In addition, the C.F.M. is proving to be of value in assessing the speed of onset, depth of anaesthesia, and length of action of drugs during studies of new anaesthetic agents. Similar monitoring may have virtues in the pharmacological evaluation of newly introduced drugs, other than anaesthetic agents, which effect the nervous system. Further, we have found monitoring of cerebral activity is of use in the assessment of patients with a variety of metabolic encephalopathies, such as those of hepatic and renal origin, during haemodialysis, and to establish the efficacy of other similar types of treatment.

We thank the physicians and surgeons at the London Hospital for permission to study patients under their care and we are grateful to Professor H. Urich for his neuropathological examinations. We warmly acknowledge the technical help of Miss S. Virden, senior E.E.G. technician, and her staff, particularly Miss M. Sully. The staff of the intensive therapy unit and the cardiac surgery theatre of the London Hospital are thanked for their help. Financial support was given by the Department of Health and Social Security and the Research Subcommittee of the Board of Governors of the London Hospital. The National Research Development Corporation sponsored the C.F.M. and Devices Limited loaned the apparatus.

United Kingdom provisional Patents Nos. 49301/67 and 8085/68 apply to the C.F.M.

\section{References}

Bickford, R. G. (1949). American fournal of Physiology, 159, 562. Binnie, C. D., Prior, P. F., Lloyd, D. S. L., Scott, D. F., and Margerison, J. H. (1970). British Medical fournal, 4, 265.

Dement, W., and Kleitman, N. (1957). Electroencephalography and Clinical Neurophysiology, 9, 673.

Efuni, S. N., Trusov, V. S., and Rabinovich, N. E. (1965). Khirurgia (Moscow), 41, 27

Feldman, S. A., and Ellis, H. (1967). Principles of Resuscitation. Oxford, Blackwell.

Fischer-Williams, M., and Cooper, R. A. (1964). Neurology (Minneapolis), $14,472$.

Haider, I., Matthew, H., and Oswald, I. (1971). Electroencephalography and Clinical Neurophysiology, 30, 23.

Maynard, D., Prior, P. F., and Scott, D. F. (1969). British Medical fournal,

Prior, P. F., and Volavka, J. (1968). Electroencephalography and Clinical Neurophysiology, 24, 593. 